\title{
Características clínicas y epidemiológicas de 25 casos de COVID-19 atendidos en la Clínica Delgado de Lima
}

\author{
Clinical and epidemiological characteristics of \\ 25 cases of COVID-19 treated at the Delgado Clinic in Lima
}

Oscar Enrique Guzmán-Del Giudice ',
Elio Paul Lucchesi-Vásquez ${ }^{2}$, Miguel Trelles-De Belaúnde ${ }^{3}$, Ramón Herbert Pinedo-Gonzales ', Marco Antonio Camere-Torrealva ${ }^{4}$, Alejandro Daly ${ }^{4} y$ Janet Elizabeth Pichilingue-Chagray ${ }^{5}$

Guzmán-Del Giudice O, Lucchesi-Vásquez E, Trelles-De Belaúnde M, Pinedo-Gonzales R, Camere-Torrealva M, Daly A y Pichilingue-Chagray J. Características clínicas y epidemiológicas de 25 casos de COVID- 19 atendidos en la Clínica Delgado de Lima. 2020;33(I): I5-24. https://doi.org/10.36393/spmi.v33i1.506

\footnotetext{
RESUMEN

Objetivo: Presentar las características clínicas y epidemiológicas de los pacientes con COVID-19 atendidos en un hospital privado de Lima. Material y Métodos: Estudio descriptivo, retrospectivo y seccional. Se presenta a los 25 casos atendidos desde el día 9 de marzo al 27 de marzo del presente año, durante la pandemia actual, que fueron atendidos en el Departamento de Emergencia de la Clínica Delgado, Miraflores, Lima. Todos ellos fueron positivos para rRT-PCR para coronavirus, realizados en el Instituto Nacional de Salud. Resultados: Casos importados, $24 \%$ o primeros contactos de ellos, $48 \%$. Doce (48\%) fueron varones. La mediana de edad fue de 38 años (rango: I I-67 años) para varones y 44 años (rango: 33-7I años) para mujeres; y una mediana global de 40 años (rango: I I-7I años). Las manifestaciones clínicas fueron: fiebre 84\%, tos seca $84 \%$, disnea $56 \%$, odinofagia $56 \%$, coriza $32 \%$, cefalea $24 \%$, dolor torácico $24 \%$, diarrea $16 \%$, mialgia $8 \%$, y fatiga $4 \%$. Las comorbilidades se hallaron en el $16 \%$ (hipertensión arterial, diabetes mellitus tipo 2, cáncer, asma e hipotiroidismo). Requirió hospitalización el 40\% (I0/25) de los casos, y sólo $8 \%$ (2/25) necesitó ser admitido a la Unidad de Cuidados Intensivos ( $\mathrm{UCI}$ ). No hubo ningún paciente fallecido. Conclusiones: Encontramos que la mayoría fueron casos importados o relacionados, no hubo diferencias en el sexo, mayor frecuencia entre la cuarta y quinta década de la vida, cuadro clínico característico (fiebre, tos y disnea), presencia de comorbilidades, menos de la mitad requirió hospitalización, hallazgos característicos en la TEM pulmonar y muy pocos requirieron ingresar a la unidad de cuidados intensivos.

Palabras claves: Coronavirus, pandemia, SARS-Cov-2, COVID-19.
}

\section{ABSTRACT}

Objective: To present the clinical and epidemiological characteristics of patients with COVID-19 treated in a private hospital in Lima. Material and Methods: A descriptive, retrospective and cross-sectional study is

I Médico asistente. Departamento de Emergencia, Clínica Delgado, Lima.

2 Médico emergenciólogo. Departamento de Emergencia, Clínica Delgado, Lima.

3 Médico radiólogo. Departamento de Radiología, Clínica Delgado, Lima.

4 Médico neumólogo. Clínica Delgado, Lima.

5 Enfermera. Responsable de Control de Infecciones, Clínica Delgado, Lima. shown. There were 25 cases attended from March 9 to March 27 this year, during the current pandemic, which were treated at the Emergency Service of the Clinic Delgado, Miraflores, Lima. All of them were positive for rRT-PCR testing for coronavirus, performed at the Instituto Nacional de Salud, Lima. Results: Imported cases, 24\% or their first contacts, $48 \%$. Twelve (48\%) were male. The median age was 38 year-old (range: 11-67 year-old) for males and 44 year-old (range: 33-71 year-old) for women; and, a global median of 40 year-old (range: 1171 year-old). Clinical manifestations were: fever $84 \%$, dry cough $84 \%$, dyspnea $56 \%$, odynophagia $56 \%$, coryza $32 \%$, headache $24 \%$, chest pain $24 \%$, diarrhea $16 \%$, myalgia $8 \%$, and fatigue $4 \%$. Comorbidities were found 
in $16 \%$ (high blood pressure, diabetes mellitus type 2, cancer, asthma and hypothyroidism). Forty per cent of cases required hospitalization, and only $8 \%$ needed to be admitted to the Intensive Care Unit (ICU). There was no deceased patient. Conclusions: We found that imported and related cases were majority, there were no differences in sex, more frequency between the fourth and fifth decade of life, characteristic clinical picture was present (fever, cough and dyspnea), comorbidities were found, less than a half required hospitalization, characteristic findings in pulmonary TEM were found and very few required entry into the ICU.

Keywords: Coronavirus, pandemic, SARS-Cov-2, COVID-19.

\section{INTRODUCCIÓN}

En diciembre del 2019, en la ciudad de Wuhan, capital de la provincia de Hubei, República Popular de China, se reportaron los primeros casos de pacientes con neumonía atípica de etiología no clara. ${ }^{1}$ Algunas semanas más adelante, lograron identificar a un nuevo betacoronavirus, similar filogenéticamente al SARS-CoV (Severe Acute Respiratory Syndrome related Coronavirus), que fue denominado en adelante SARS-CoV-2.,3

Antes del año 2019 se habían identificado seis tipos de coronavirus causantes de enfermedades en humanos. Cuatro producen enfermedades respiratorias habitualmente leves, caracterizadas por síntomas en la vía respiratoria superior como un resfriado común; $y$, dos betacoronavirus causan enfermedades más graves: el SARS-COV, descubierto en el año 2002 y el MERS-COV (Middle East Respiratory Syndrome related Coronavirus), descubierto en el año 2012.

El gobierno chino intentó contener este patógeno a través del aislamiento completo de la mencionada provincia y realizando seguimiento epidemiológico de todos los contactos para llevarlos a una cuarentena obligatoria. Sin embargo, ante la aparición de nuevos casos en varios países del mundo y su incremento exponencial producidos por el mismo agente infeccioso, la Organización Mundial de la Salud (OMS) declaró la situación como pandemia el 11 de marzo del 2020. La enfermedad relacionada al SARSCoV-2 fue denominada COVID-19 (Coronavirus Disease 2019), manifestada fundamentalmente por el compromiso respiratorio neumónico, intersticial y multifocal, acompañado de un cortejo sintomático diverso que puede incluir fiebre, tos, disnea, coriza, diarrea, cefalea, anosmia, etc., y el eventual compromiso de otros sistemas.

En el Perú, hasta la fecha de las atenciones de los casos que se reportan, el único ente autorizado para el diagnóstico de este nuevo virus era el Instituto Nacional de Salud (INS), perteneciente al Ministerio de Salud (MINSA). Esta institución realiza el diagnóstico a través de pruebas moleculares rRT-PCR para SARS-CoV-2 provistas por la OMS. El primer caso en territorio peruano confirmado por el MINSA fue dado a conocer el día 6 de marzo del $2020{ }^{4}$ En la ciudad de Lima, el distrito de Miraflores tiene la segunda mayor población flotante más grande de todo el país luego de la ciudad de Cuzco. Se estima que la población flotante de Miraflores bordea las 500000 personas diariamente, con una actividad comercial y turística muy intensa. ${ }^{5}$ Esta singular característica del distrito la convierte en un área geográfica potencialmente vulnerable a cualquier enfermedad infecciosa en la que la población flotante puede ser el vector y factor más relacionado a la diseminación de estas enfermedades.

La Clínica Delgado es un hospital privado de alto nivel de complejidad ubicado en el distrito de Miraflores y se ha constituido en una institución referente a nivel local, nacional e internacional de la región sur de las Américas, atendiendo pacientes nacionales y extranjeros.

Es en este contexto, el reporte temprano de los casos atendidos en este nosocomio puede ser de utilidad para orientar y presentar las características del COVID-19 en la realidad peruana.

\section{MARTERIAL Y MÉTODOS}

Se realizó un estudio descriptivo, retrospectivo y seccional sobre 25 casos atendidos desde el día 09 de marzo del 2020, en que se detectó nuestro primer caso, hasta el día 27 de marzo del mismo año. El corte de tiempo ha sido discrecional. El interés de los autores fue lograr una casuística relevante que pueda ser reportada tempranamente para la divulgación dentro de la comunidad médica peruana, con la esperanza que nuestra experiencia ayude en la atención de futuros pacientes en los diferentes servicios de emergencia del país y de la región sur de las Américas.

Todos los casos fueron declarados positivos para SARSCoV-2 a través de la prueba molecular de rRT-PCR realizada por el INS. Cabe resaltar que, para la fecha de la atención de estos pacientes, no se disponía de protocolos nacionales establecidos, ni consenso para el tratamiento y manejo de la enfermedad. Sólo había recomendaciones disímiles que provenían del extranjero, algunos reportes anecdóticos o de cohortes muy pequeños, sin la fuerza suficiente como para sustentar un nivel de evidencia alto. Tampoco se disponía de pruebas serológicas rápidas u otras técnicas de diagnóstico autorizadas por la autoridad sanitaria del MINSA.

\section{RESULTADOS}

Durante el periodo de observación se tuvo 25 pacientes (Gráfica 1). Doce (48\%) fueron varones; y, la mediana de edad fue de 38 años (rango: 11-67 años) para varones y 44 años (rango: 33-71 años) para mujeres; y una mediana global de 40 años (rango: 11-71 años). (Gráfica 2).

Los síntomas más frecuentes fueron; fiebre, tos seca, disnea y odinofagia (Gráficas 3 y 4).

Entre los exámenes auxiliares, los promedios de saturación arterial de oxígeno $\left(\mathrm{SatO}_{2}\right)$, cuenta total de linfocitos, transaminasas fueron normales. El promedio de proteína $\mathrm{C}$ reactiva estuvo elevado. (Tabla 1 ).

La radiografía de tórax solo fue tomada en 3 casos, siendo una anormal (opacidades en ambos campos pulmonares) Figura 1. 
Gráfica I. Número de casos diagnosticados en el Departamento de Emergencia, Clínica Delgado, Lima, 09 de marzo-27 de marzo del 2020.

Número de casos diagnosticados por día $=$ Acumulado de casos diagnosticados

- Línea de tendencia de Acumulado de casos diagnosticados $\mathrm{R}^{2}=0,987$

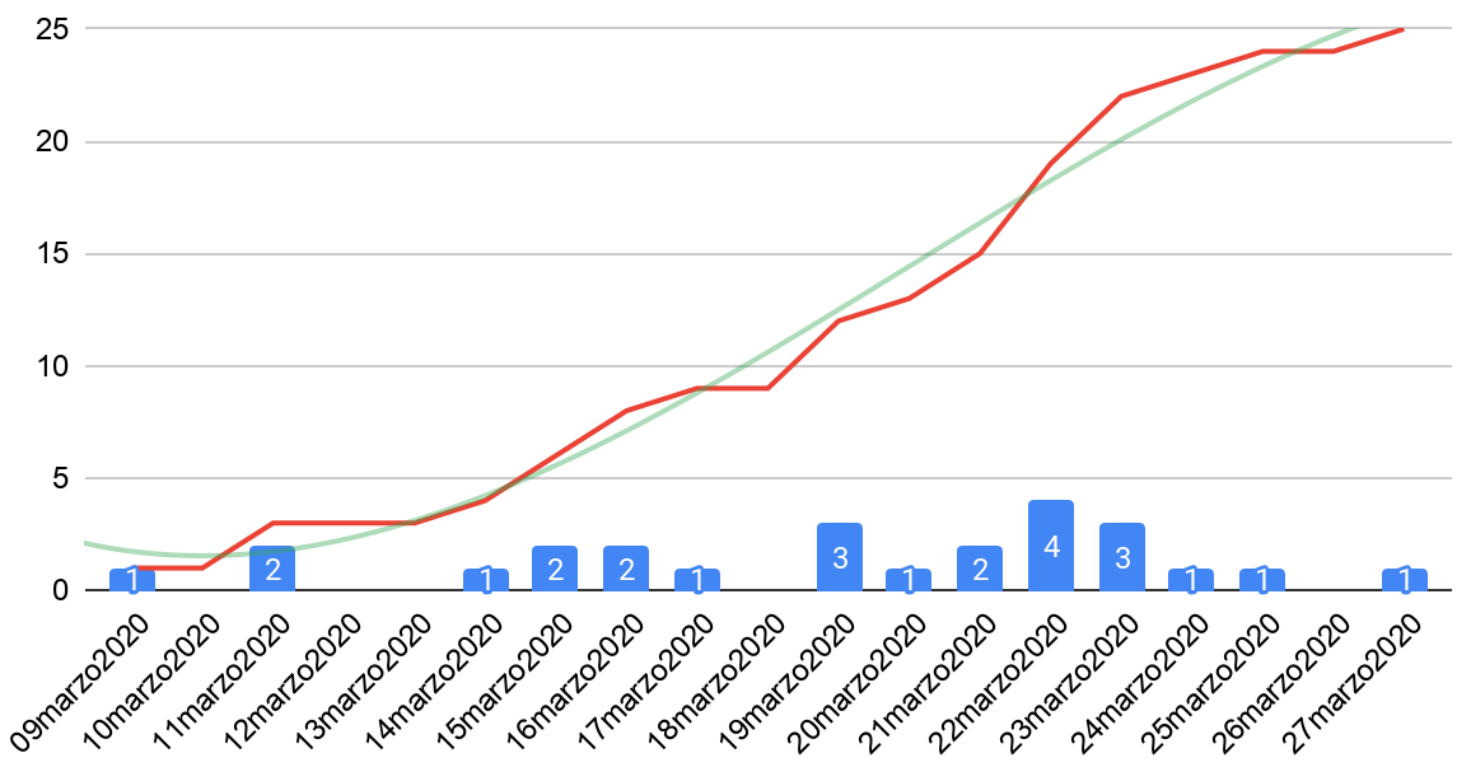

Gráfica 2. Dispersión y mediana por sexo.

Dispersión y mediana de edad por sexo

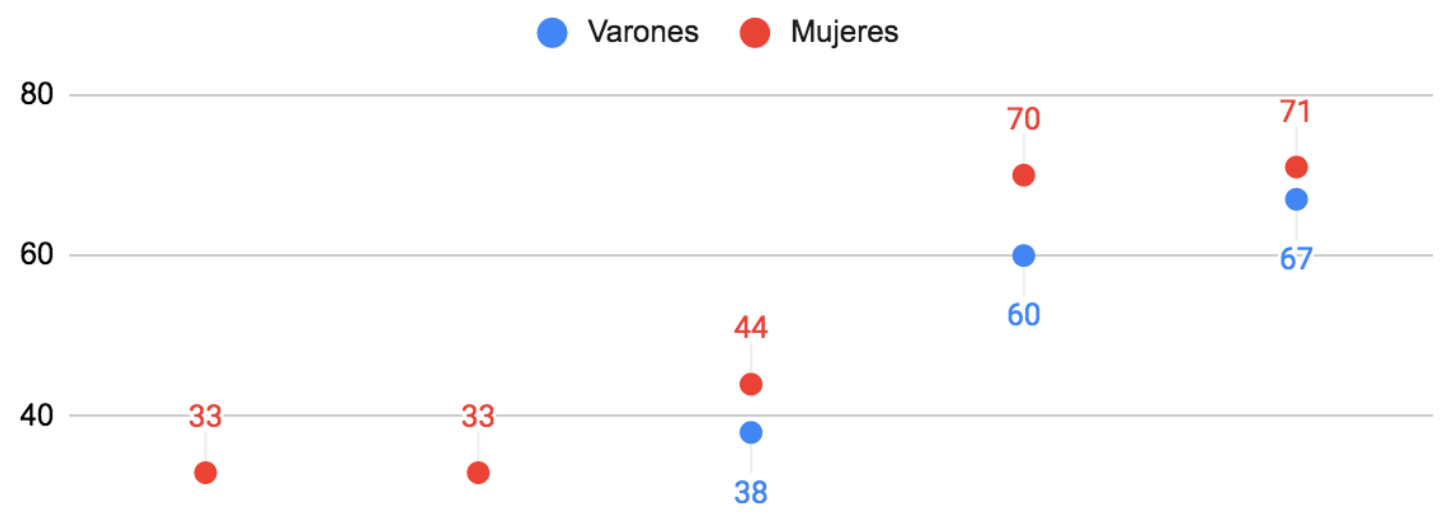

20

16

$0 \frac{11}{\text { Mínimo }}$

Percentil 3

mediana

Percentil 97

Máximo

La tomografía espiral multicorte (TEM) de tórax y pulmones sin contrastef fue realizada en 14 pacientes y en todos los casos fueron anormales, describiéndose consolidaciones múltiples en los campos pulmonares, con aspecto de vidrio deslustrado (ground glass) y/o "piso loco" (crazy paving), opacidades redondeadas. Las lesiones usualmente fueron bilaterales, periféricas, subpleurales y basales. (Figuras 2-7).

Las comorbilidades se presentaron en el 16\% (4/25 casos: hipertensión arterial (HTA), diabetes mellitus tipo 2 (DM2), cáncer, asma e hipotiroidismo).

Diez pacientes (40\%) requirieron hospitalización, y sólo 
Gráfica 3. Frecuencia de los signos y síntomas, COVID-I9.

\section{Frecuencia de Signos y Síntomas (n:25)}

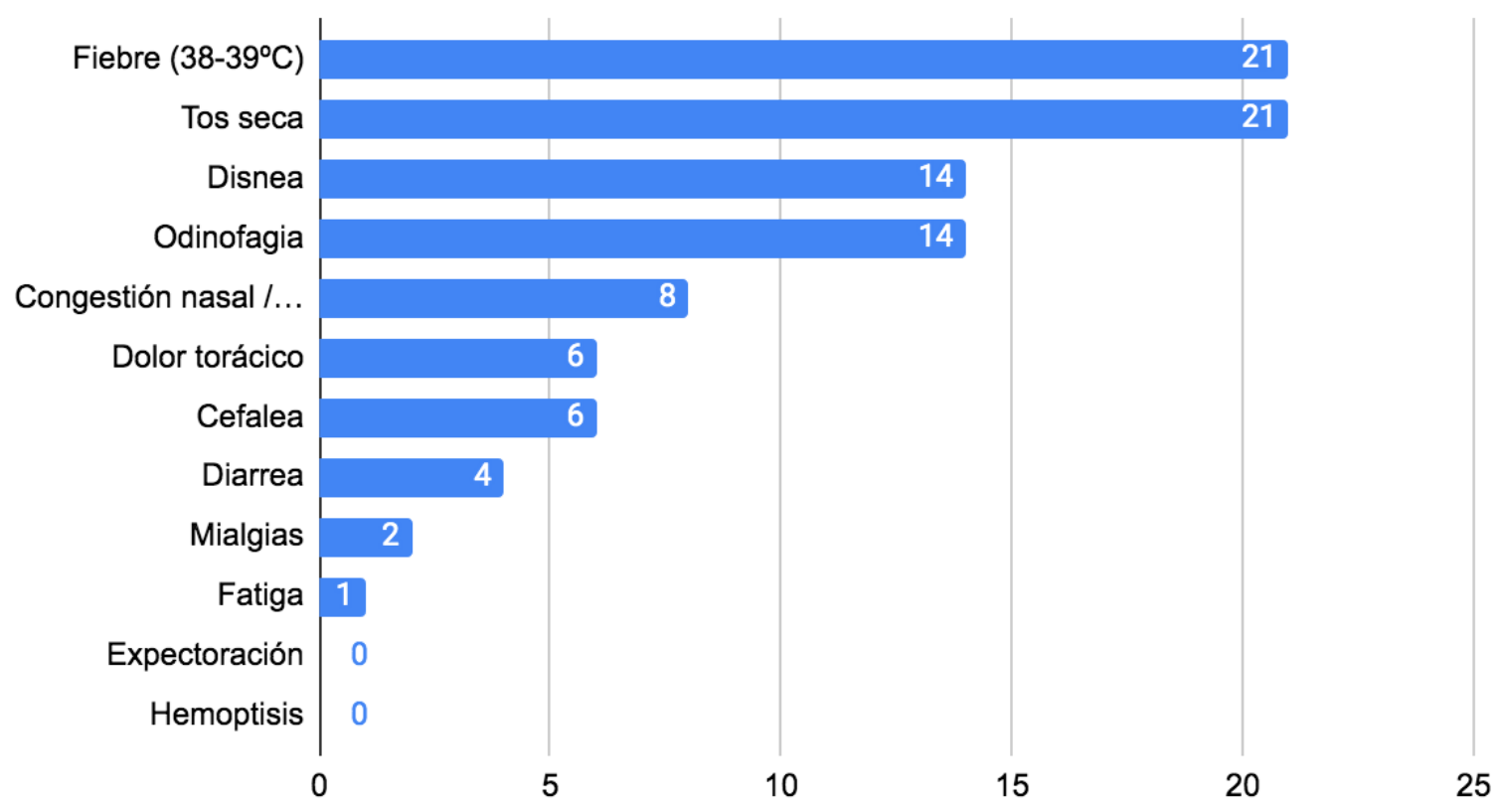

Gráfica 4. Frecuencia de signos y síntomas según el sexo.

Frecuencia de signos y síntomas por sexo (n:25)

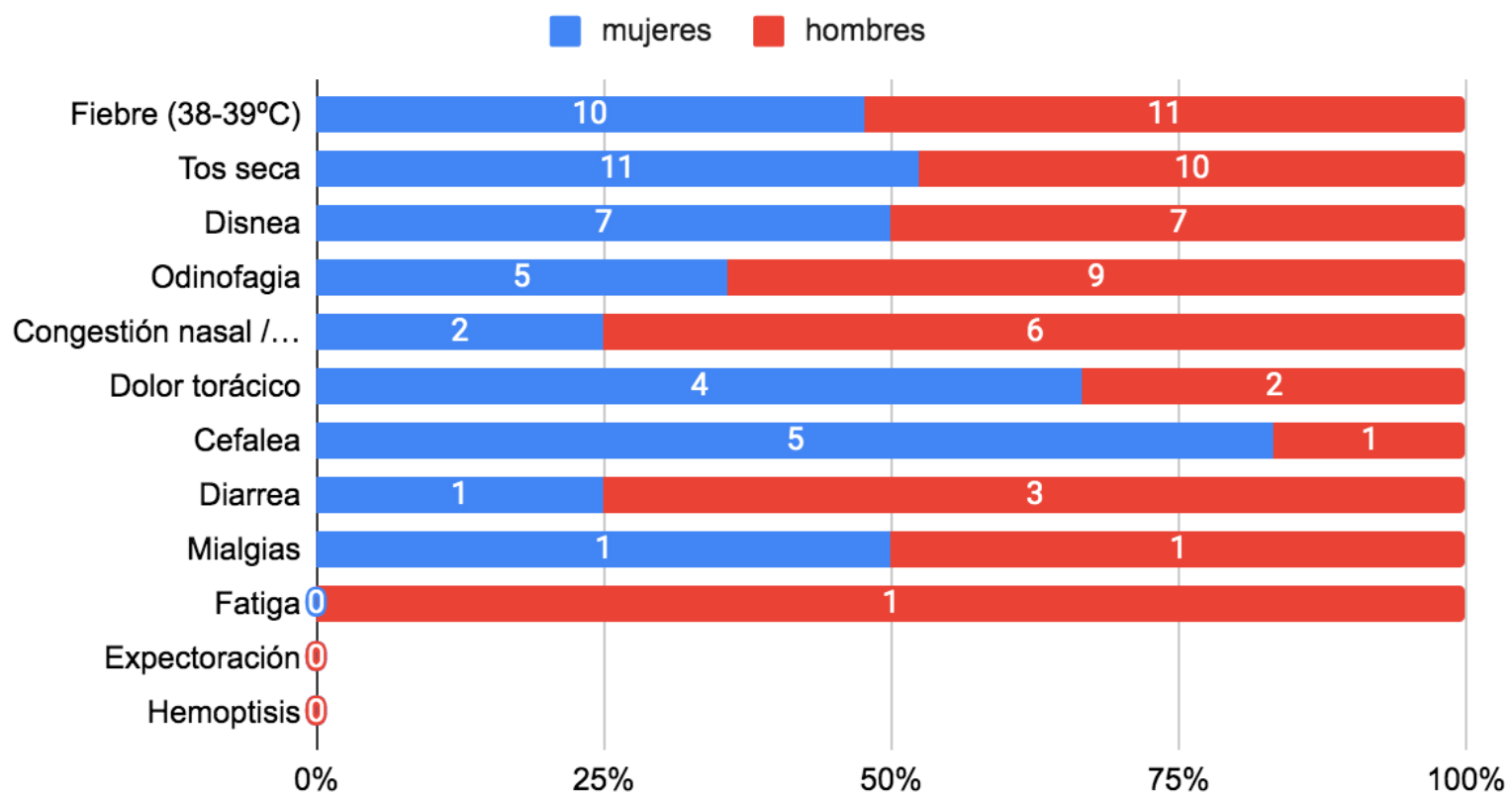

dos (8\%) fueron admitidos a la Unidad de Cuidados Intensivos (UCI).

La gran mayoría correspondió a casos importados (24\%)

o a los primeros contactos de ellos en territorio peruano (48\%). (Gráfica 5).
Los diagnósticos iniciales fueron infección respiratoria alta, neumonía y bronconeumonía donde el antecedente epidemiológico ayudó apara la sospecha de COVID-19. Ocho pacientes $(32 \%)$ fueron hospitalizados en la sala de aislados, dos (8\%) paso a UCI y el resto $(60 \% 9$ fue 
Tabla I. Exámenes auxiliares. Pacientes con COVID-19, Clínica Delgado, Lima.

$\begin{array}{llllll} & \mathrm{SatO}_{2}(\%) & \text { Linfocitos } / \mathrm{mm}^{3} & \mathrm{TGP}(\mathrm{U} / \mathrm{L}) & \mathrm{TGO}(\mathrm{U} / \mathrm{L}) & \text { Proteína C reactiva }(\mathrm{mg} / \mathrm{L}) \\ \mathrm{n} / 25 & 25 & 17 & 7 & 7 & 17 \\ \text { promedio } & 97 & 1723,4 & 45 & 32 & 3,54 \\ \text { mínimo } & 88 & 928,0 & 13 & 16 & 0,04 \\ \text { máximo } & 100 & 2498,7 & 125 & 81 & 14,5\end{array}$

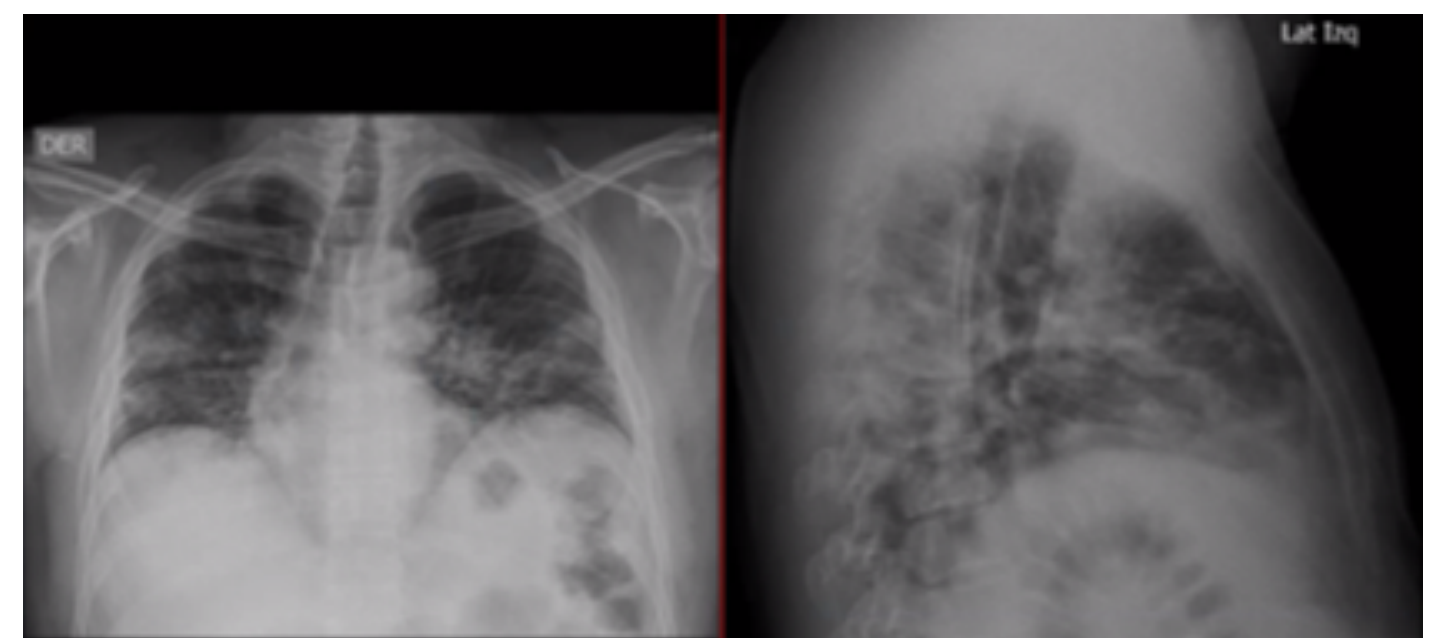

Figura I. Radiografía de tórax. COVID-I9.

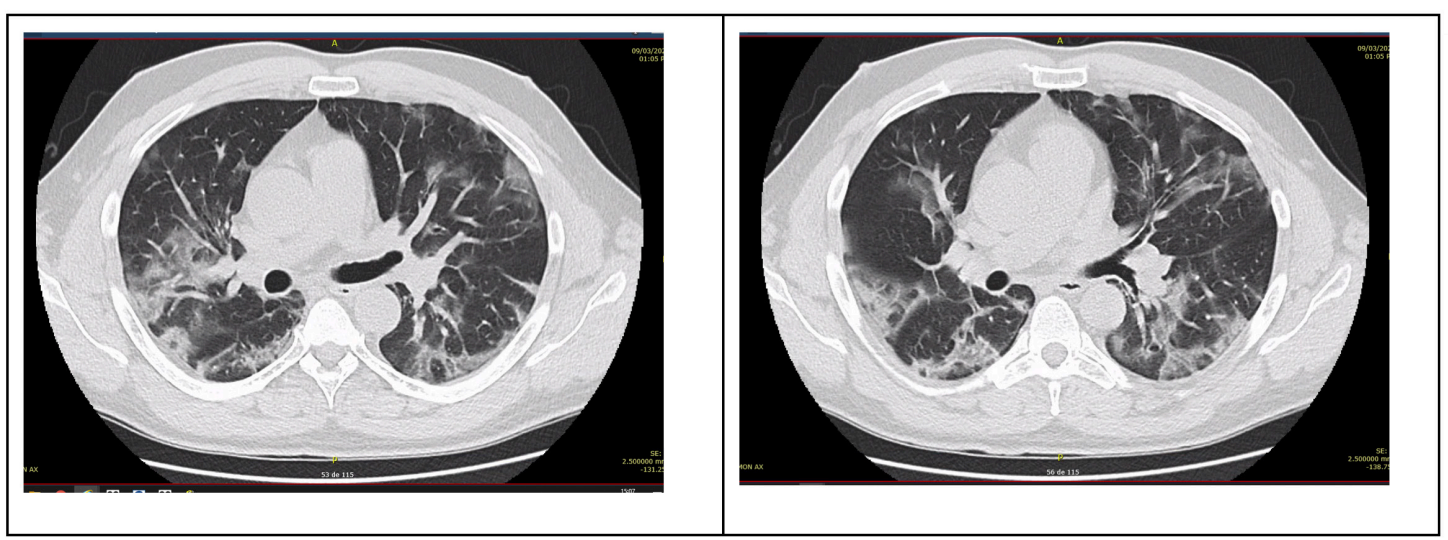

Figura 2. COVID-I9.
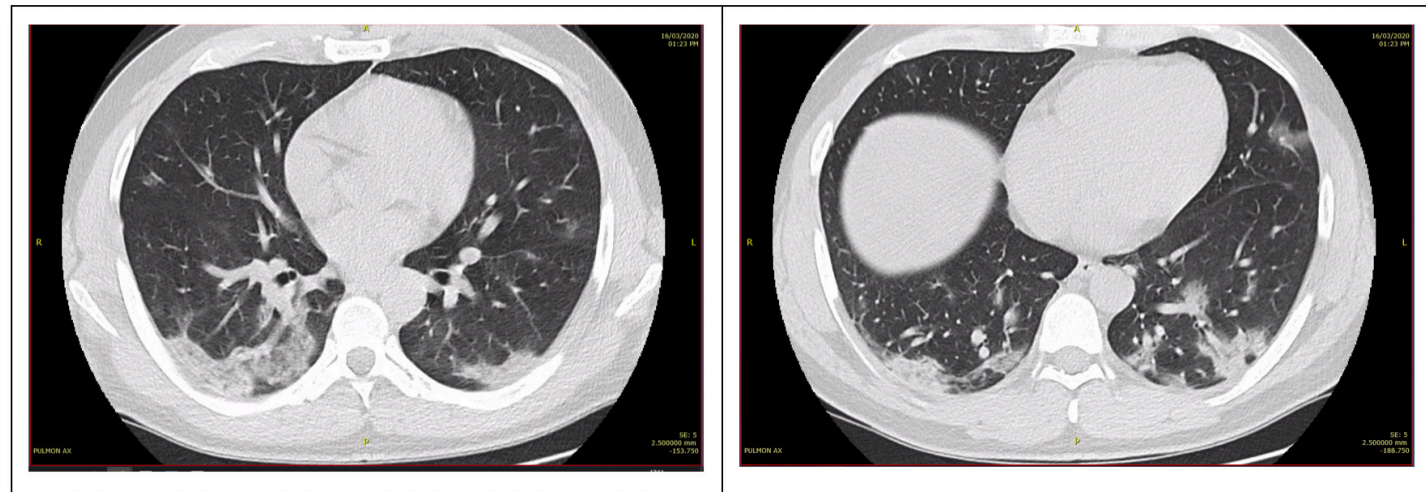

Figura 3. COVID-19. 

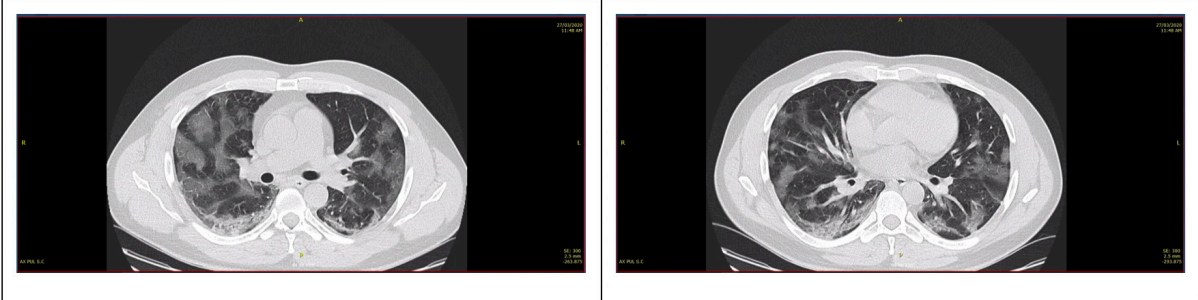

Figura 4. COVID-19.
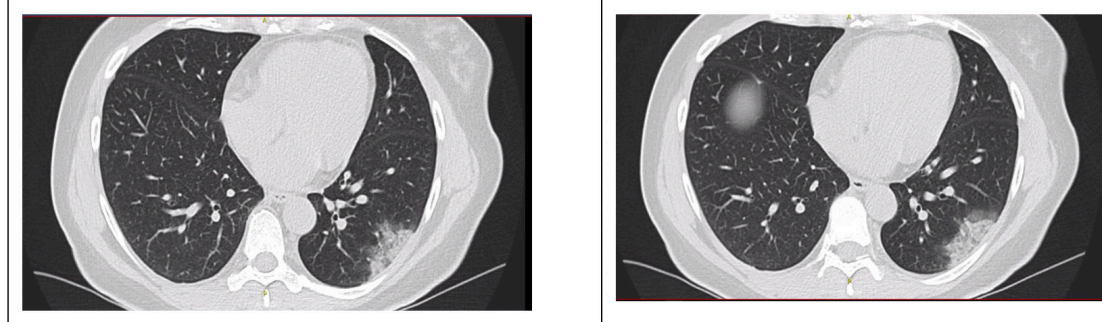

Figura 5. COVID-19.
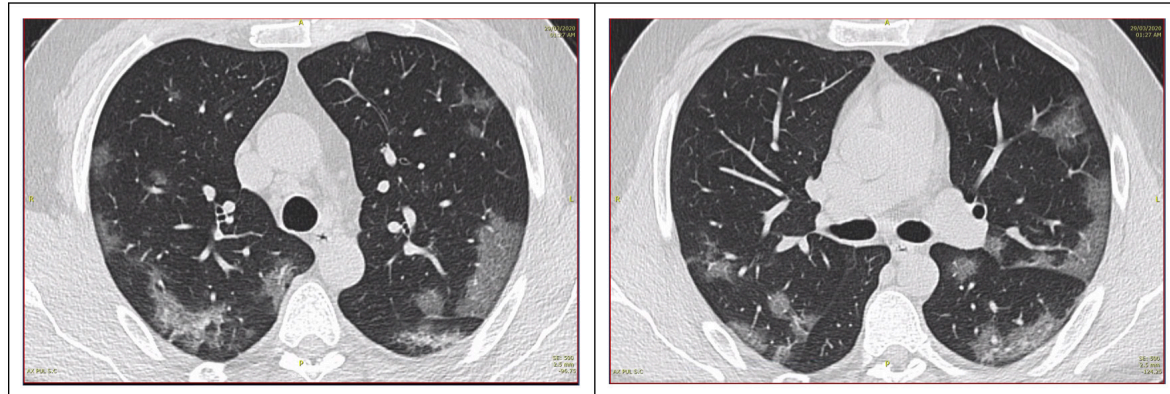

Figura 6. COVID-19.
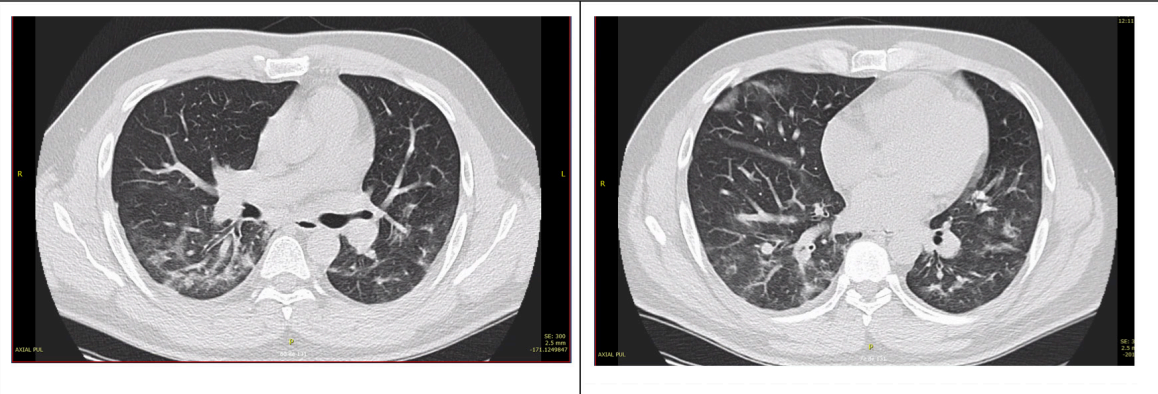

Figura 7. COVID-19.

manejado ambulatoriamente con las recomendaciones de aislamiento en su domicilio.

El tratamiento fue sintomático y de soporte según el caso, no habiendo todavía una definición en cuanto a terapia establecida para COVID-19.

\section{DISCUSIÓN}

Los coronavirus $(\mathrm{CoV})$ son una familia de virus ARN monocatenarios que habitualmente afectan a otras especies diferentes a la humana (camélidos, vacas, felinos, murciélagos), en quienes producen enfermedades respiratorias, entéricas, hepáticas y neurológicas. La transmisión de estas cepas hacia los humanos se da por el contacto directo o indirecto con los animales.

Dentro de esta gran familia, se han identificado a la fecha siete coronavirus que tienen la capacidad de infectar a los humanos (HCoV):

CoV humanos comunes: HCoV-229E y HCoV-NL63 (alphaCoVs); HCoV-OC43 y HCoV-HKU1 (betaCoV del linaje A), responsables de aproximadamente 5 a $10 \%$ de los resfriados comunes. 


\section{Gráfico 5. Historia o antecedente de un nexo epidemiológico}

\section{Nexo epidemiológico}

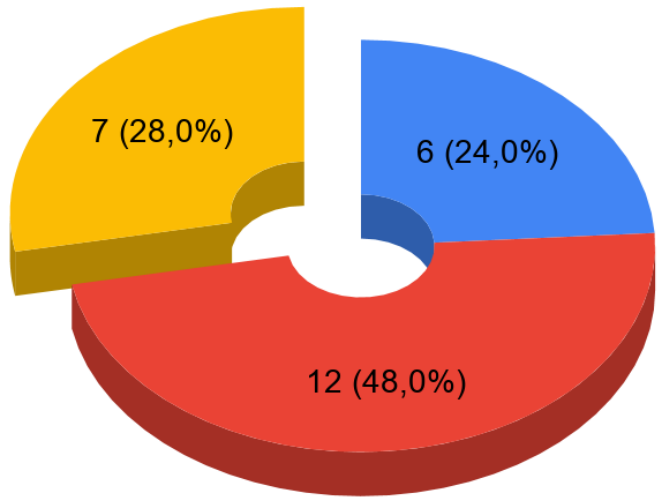

Viaje al extranjero en los últimos 14 días

Subgrupo de $\beta$-coronavirus (casos graves epidémicos): MERS-CoV, SARS-CoV, y el actual SARS-CoV-2.

Se sugiere que el origen de la enfermedad COVID-19 es zoonótica. ${ }^{6}$ El SARS-CoV-2 comparte siete proteínas no estructurales con el virus causante del SARS-CoV (82\% de identidad de nucleótidos con el del SARS-CoV). En la actualidad se describe que este virus utiliza el mismo mecanismo utilizado por el SARS-CoV para penetrar a las células, a través del receptor de la enzima convertidora de angiotensina II (ACE2). ${ }^{\text {? }}$

El cuadro clínico producido por el SARS-CoV-2 es variable, pero fiebre, tos seca ty disnea son las manifestaciones más frecuentes en todas las series. ${ }^{8-11}$ Esto también se encontró en nuestra serie. Gráficas 3 y 4.

\section{Perfil epidemiológico}

De acuerdo a la tabla abajo mencionada, el perfil epidemiológico más frecuente es una persona entre los 40 a 60 años, de sexo masculino, en los que aproximadamente la tercera parte de ellos presenta comorbilidades. Se ha reportado que la mayor mortalidad hospitalaria está asociada a pacientes ancianos, un puntaje elevado en el score secuencial SOFA y la elevación del dímero D mayor a $1 \mu \mathrm{g} / \mathrm{mL}$, hallazgos conocidos como factores de riesgo asociados a neumonía severa. ${ }^{12}$

\section{Técnicas de diagnóstico}

\section{Hallazgos de laboratorio general}

Alrededor de $80 \%$ de los pacientes tiene linfopenia con recuento celular de glóbulos blancos normal o casi normal. ${ }^{13}$ Se ha observado que la progresión de la linfopenia es un factor pronóstico individual de mortalidad. Los pacientes que requieren ingreso a UCI tienden a evolucionar hacia un recuento de linfocitos menor al 5\% del recuento total. ${ }^{14}$ En nuestra corta serie no fue frecuente la linfopenia.
También se puede encontrar que los pacientes tienden a tener una elevación de los marcadores inflamatorios. Una revisión de casos muestra que los niveles altos de proteína $\mathrm{C}$ reactiva (PCR) se relacionaron con mayor necesidad de oxigenoterapia. Se ha descrito que entre los 18 pacientes hospitalizados de una cohorte con infección confirmada por SARS-COV2, los pacientes que tuvieron necesidad de oxígeno suplementario tuvieron una media de PCR de $65,6 \mathrm{mg} / \mathrm{dl}$, en comparación con aquellos que no necesitaron oxígeno, quienes presentaron una media de $11,1 \mathrm{mg} / \mathrm{dl} .{ }^{15} \mathrm{En}$ nuestra serie, sí fue frecuente hallar una PCR incrmentada. La procalcitonina no suele tener una elevación significativa en estos pacientes. Sin embargo, una elevación de ella ha sido asociada a cinco veces mayor riesgo de infección grave. ${ }^{16}$

Algunos otros hallazgos comúnmente encontrados en estos pacientes y relacionados con enfermedad muy severa, mayor mortalidad $\mathrm{y}$, por ende, peor pronóstico fueron elevación de la ferritina, deshidrogenasfa láctica (DLH) mayor a $245 \mathrm{U} / \mathrm{L}$, relación neutrófilos/linfocitos (RNL) mayor a 3 y elevación de troponinas. ${ }^{17,18}$

\section{Técnicas moleculares}

Al momento de esta publicación, el diagnóstico molecular confirmatorio se realiza a través de la identificación del virus SARS-CoV-2 por técnica de Reacción en Cadena de la Polimerasa en tiempo real (RT-PCR), mediante el secuenciamiento parcial o total del genoma viral. ${ }^{19}$ Esta prueba confirmatoria es altamente específica; sin embargo, tiene una sensibilidad relativamente baja, cercana al 60 a $70 \%$. Por ende, es posible tener un número significativo de casos falso negativo. ${ }^{20}$

Las razones por las que puede existir una baja eficiencia de estas pruebas podrían ser variación en la tasa de detección de diferentes fabricantes, baja carga viral del paciente y 
muestreo clínico incorrecto. Por otro lado, esta prueba sólo es realizada por el INS, a donde deben remitirse todas las muestras de sangre.

\section{Tomografía espiral multicorte (TEM) de tórax y pulmones sin contraste}

En la TEM de tórax y pulmones sin contraste de los pacientes adultos se puede encontrar: opacidades tipo vidrio esmerilado (bilaterales, subpleurales, periféricas), patrón de "piso loco" (crazy paving) con opacidades tipo vidrio esmerilado y engrosamiento inter e intra lobular septal, consolidación de los espacios aereados, engrosamiento broncovascular y bronquiectasias traccionadas ${ }^{21-25}$

Elpatrón en vidrio esmeriladoylasopacidades consolidativas usualmente son bilaterales, periféricas y de distribución basal. $^{26,} 27$ Por otro lado, estos hallazgos se describen también en pacientes asintomáticos u oligosintomáticos. En un estudio retrospectivo, en 112 pacientes asintomáticos, se encontró que el 54\% tenía cambios en la tomografía. ${ }^{28}$ Por esta razón, algunas publicaciones sugieren que la TEM de tórax y pulmones sin contraste tendría una alta sensibilidad para la detección de casos sospechosos, pudiendo convertirse en una herramienta útil para las áreas críticas y de cuidado agudo como un servicio de emergencia. ${ }^{29}$

La peculiar distribución y características de las lesiones ha hecho que muchas guías clínicas y protocolos de manejo internacionales adopten la tomografía de tórax como una técnica de tamizaje o cribado inicial en casos sospechosos de infección por SARS-CoV-2. Tal fue el caso de las denominadas "clínicas de fiebre" existentes en la ciudad de Wuhan de la República Popular China. ${ }^{30}$ También, en un estudio comparativo y retrospectivo de 51 casos, realizado en el Centro Médico Taizhou Enze, China, entre enero y febrero pasado, con respecto a la sensibilidad de la TEM de tórax y pulmones versus la RT-PCR, se encontró que la sensibilidad de la TEM fue mayor que la de RT-PCR (98\% vs $71 \%$, respectivamente).$^{31}$

La radiografía de tórax tiene poca sensibilidad y a veces solo muestra cambios inespecíficos. En los casos en que realizamos TEM, el cien por ciento mostró las alteraciones que sugieren COVID-19.

\section{Ecografía en el punto de atención}

Se ha observado que los hallazgos ecográficos POCUS (point of care ultrasound) de pulmón se correlacionan bien con los hallazgos encontrados en la TEM de tórax para pacientes con infección por SARS-CoV-2, pudiendo utilizarse esta técnica para un tamizaje o cribado rápido en pacientes que acuden a la emergencia con síntomas respiratorios bajos, o en condiciones en las cuales no exista acceso a la TEM de tórax. ${ }^{32}$ Además, también tendría la ventaja de realizarse al lado del paciente, sin exposición a radiación, con la posibilidad de repetición, evitando la movilización del paciente y disminuyendo el riesgo de contaminación nosocomial durante del transporte. Así mismo, el POCUS también puede ayudar a determinar diagnósticos alternativos que expliquen la condición del paciente con alta sensibilidad y especificidad.

La alta sensibilidad del ultrasonido se fundamentaría en que el mayor compromiso observado en COVID-19 se produce por la inflamación del intersticio pulmonar subpleural y periférico, que incrementa el contenido de agua en esta zona. Por esta razón es fácilmente accesible con el transductor ecográfico. Los patrones típicos son la visualización de las líneas B1 y B2 y alteraciones en el aspecto de la línea pleural. Con la progresión de la enfermedad y posterior compromiso de los septos interalveolares y los alvéolos se puede observar patrones de consolidación. No realizamos estudios de ultarsonido.

La enfermedad producida por la infección del SARSCoV-2 es nueva en el Perú y los primeros casos que hemos atendido han presentado un cuadro clínico similar a los reportados en China y otras latitudes. En nuestra serie no tuvimos una predominancia significativa en la distribución por sexo pero sí notamos una discreta diferencia en la edad de presentación entre ambos sexos, con una mediana de 38 años para varones y 44 años para mujeres.

Las comorbilidades se presentaron en el 16\% (hipertensión arterial (HTA), diabetes mellitus tipo 2, cáncer, asma e hipotiroidismo).

Requirió hospitalización el 40\% de los casos, y sólo 8\% necesitó ser admitido a la Unidad de Cuidados Intensivos (UCI) por lo que podemos afirmar que nuestra serie fue de casos leves y moderados.

La gran mayoría correspondió a casos importados (24\%) o a los primeros contactos de ellos (48\%). Este hallazgo correlaciona con el área de influencia geográfica de la clínica, que se encuentra dentro del distrito limeño con mayor población flotante y el perfil de usuarios habituales del nosocomio, esto es turistas, viajeros frecuentes, etc.

Desde el punto de vista clínico, es importante resaltar que la mayoría de pacientes no tuvo hallazgos importantes en la exploración física. La mayor parte de ellos tuvieron ruidos respiratorios normales o casi normales. Los hallazgos clínicos que correlacionan con una neumonía típica (soplo tubario, crépitos, etc.) estuvieron ausentes en casi todos los pacientes, incluso en aquellos en los que se observó un gran compromiso pulmonar a través del TEM pulmonar sin contraste. Sin embargo, en aquellos que estuvieron más comprometidos, el aspecto más relevante fue la presencia de disnea y fiebre persistente, asociada al incremento de la frecuencia respiratoria, del trabajo respiratorio y/o una disminución en la saturación de oxígeno con respecto a su basal. También es importante resaltar que se observó disociación clínico-radiológica, pudiendo encontrarse un gran compromiso pulmonar con pobre sintomatología correspondiente y viceversa.

En tanto no se disponía de scores predictivos para esta enfermedad y los hallazgos en los estudios complementarios habitualmente no mostraban mayores cambios o existía una disociación marcada, el criterio y el juicio clínico fueron los mayores puntos de apoyo para la disposición de los pacientes. Por este motivo, muchos de los casos fueron dados de alta, con la instrucción de mantenerse 
en cuarentena y aislamiento domiciliario. Todos fueron instruidos para observar su evolución clínica y para buscar una reevaluación médica frente a la aparición de cualquier signo de alarma. Aprendimos que la evolución tórpida de algunos de ellos se suscitó en corto tiempo.

Recomendamos ser inquisitivos en la búsqueda de los factores de riesgo biológico y social que pudieran limitar una correcta atención domiciliaria.

La TEM de tórax y pulmones sin contraste demostró tener una sensibilidad muy alta para el diagnóstico, comparada contra el 60-70\% de sensibilidad de la PCR-RT para SARS$\mathrm{CoV}-2$; convirtiéndose en una herramienta útil y rápida para apoyar y acotar el diagnóstico etiológico. Del total de todos nuestros pacientes positivos para SARS-COV-2, el 100\% de los que se les practicó una TEM de tórax y pulmones sin contraste tuvo hallazgos radiológicos compatibles con COVID-19.

En nuestra experiencia, esta técnica nos ha permitido identificar de manera muy eficiente y rápida a los pacientes con alto grado de probabilidad diagnóstica, y aportar activamente en el cerco epidemiológico necesario para contener la pandemia producida por este virus dentro del Perú. Por este motivo, alentamos la realización de la misma en las instituciones que tengan esta facilidad ante la sospecha clínica, en los pacientes vulnerables con antecedentes de riesgo en los que se sospeche COVID-19 y/o lugares donde no cuente con la capacidad de diagnóstico con PCR-RT o pruebas rápidas para SARS-CoV-2.

La radiografía de tórax podría ser útil en lugares donde no exista la facilidad para realizar una TEM de tórax y pulmones. Sin embargo, es necesario puntualizar que tiene una baja tasa de sensibilidad, y por ende una alta tasa de falsos negativos. Esto es particularmente válido en los estadios iniciales de la enfermedad. El ultrasonido pulmonar podría constituirse en una herramienta de diagnóstico útil y rápido, incluso en establecimientos de salud que cuenten con facilidades para obtener una tomografía de tórax y pulmones.

Consideramos que los casos presentados fueron formas leves y moderadas de COVI-19 por lo que solo dos fueron admitidos a UCI pero no ocurrió muerte alguna.

Finalmente, en tanto es una enfermedad nueva, y de la que estamos aprendiendo día a día, recomendamos guiarnos por el criterio y razonamiento clínico, apoyándonos en los estudios auxiliares que, ya lo sabemos, podrían tener una marcada disociación con los hallazgos observables en el paciente.

\section{CONCLUSIONES}

En nuestra serie de pacientes con COVID-19 encontramos características clínicas similares a las descritas en las primeras series de casos: sin diferencias en el sexo, mayor frecuencia entre la cuarta y quinta década de la vida, presencia de comorbilidades, menos de la mitad requirió hospitalización y muy pocos requirieron ingresar a la unidad de cuidados intensivos. y hallazgos característicos en la TEM pulmonar.

\section{REFERENCIAS BIBLIOGRÁFICAS}

I. Huang C, Wang Y, Li X, et al. Clinical features of patients infected with 2019 novel coronavirus in Wuhan, China. Lancet. 2020;395:497-506.

2. Lu R, Zhao X, Li J, et al. Genomic characterization and epidemiology of 2019 novel coronavirus: implications for virus origins and receptor binding. Lancet. 2020;395:565-574.

3. Zhu N, Zhang D, Wang W, et al. A novel coronavirus from patients with pneumonia in China, 2019. N Engl J Med. 2020;382:727-733.

4. Nota de prensa: https://www.gob.pe/institucion/minsa/ noticias/I 08937 -minsa-proceso- I 55 -muestras-porcoronavirus-covid-19-y-una-resulto-positivo

5. Blog: https://mirafloresmiraflores.blogspot.com/20I I/0I/demis-continuos-paseos-por-las-calles.html

6. Yong-Zhen. A Genomic perspective on the origing and emergence of SARS- COV-2. Cell. Published: March 26, 2020. https://doi.org/10.1016/j.cell.2020-03-035

7. Zhou P, et al. A pneumonia outbreak associated with a new coronavirus of probable bat origin. Nature.2020;10.1038/ s4I586-020-20I2-7

8. Cheng $\mathrm{N}$, et al. Epidemiological and clinical characteristics of 99 cases of 2019 novel coronavirus pneumonia in Wuhan, China: a descriptive study. Lancet. 2020; 395: 507-13

9. Huang C, Wang Y, Li X, Ren L, Zao J, Hu Y, et al. Clinical features of patients infected with 2019 novel coronavirus in Wuhan, China. Lancet. 2020; 395: 497-506

10. Wang, Jian-ming; Liu, Ji-yang; Chen, Zhong; Li, Gang; Zheng, Zhi-jian; Qiu, Shao-qin; et al. Clinical Characteristics of Coronavirus Disease 2019 in China. N Engl J Med. February 28, 2020. doi: I0.1056/NEJMoa2002032

II. Wang D, Hu B, Hu C, Zhu F, Liu X, Zhang J, et al. Clinical characteristics of 138 hospitalized patients with 2019 novel coronavirus-infected pneumonia in Wuhan, China. JAMA. 2020 Feb 7; 323(II):106I-1069. doi: 10.100I/ jama.2020.1585. [Epub ahead of print]

12. Zhou $\mathrm{F}$ et al. Clinical course and risk factors for mortality of adult inpatients with COVID-19 in Wuhan, China: a retrospective cohort study. Lancet. 2020; (published online March 9.)

13. Zhang J], Dong $X$, Cao YY, Yuan YD, Yang YB, Yan YQ et al. Clinical characteristics of 140 patients infected whit SARSCoV-2 in Wuhan, China. Allergy. 18 febrero 2020. doi: 10.1 I I I//all.13238 https://onlinelibrary.wiley.com/doi/ full/I0.1III/all. 14238

14. Li Tan, Qi Wang, Duanyang Zhang, Jinya Ding, Qianchuan Huang, Yi-Quan Tang, et al. Lymphopenia predicts disease severity of COVID-19: a descriptive and predictive study, China. Signal Transduction and Targeted Therapy. (2020) 5:33. doi: 10.1038/s41392-020-0138-4 https://www.nature. com/articles/s41392-020-0148-4.pdf

15. Young BE, Ong SWX, Kalimuddin S, Low JG, Tan SY, Loh J, et al. Epidemiologic features and clinical course of patients infected with SARS-CoV-2 in Singapore. JAMA. Published online March 3, 2020. doi:10.100I/jama.2020.3204.

16. Lippi G, Plebani M. Procalcitonin in patients with severe coronavirus disease 2019 (COVID-19): A metanalysis. Clinica Chimica Acta. 2020; 505:190-191.Disponible en:https://www.sciencedirect.com/science/article/pii/S00098 98/20301066?via\%3Dihub

17. Zhou B, She J,Wang J, Ma X. Utility of ferritin, procalcitonin, and $C$-reactive protein in severe patients with 2019 novel 
coronavirus disease. BMC Pulmonary Medicine. 19 mar 2020. URL disponible en: https://www.researchsquare.com/ article/rs- |8079/v|

18. Jingyuan Liu, Yao Liu, Pan Xiang, Lin Pu, Haofeng Xiong, Chuansheng Li, Ming Zhang, Jianbo Tan, et al. Neutrophilto-lymphocyte ratio predicts severe illness patients with 2019 novel coronavirus in the early stage. Critical Care Medicine Department. Ditan Hospital, Capital Medical University Beijing, China. Feb I2, 2020. BMJ Yale. doi: https:// doi.org/I0.1 I0I/2020.02.10.20021584.

19. Documento Técnico Atención y Manejo Clínico de Casos de COVID-19. Aprobado por la Resolución Ministerial $\mathrm{N}^{\circ}$ 084-2020-MINSA.

20. Tao Ai, Zhenlu Yang, Hongyan Hou, Chenao Zhan, Chong Chen, Wenzhi, Qian Tao, Ziyong Sun, Liming Xia. Correlation of chest CT and RT-PCR testing in coronavirus disease 2019 (COVID-19) in China: A report of 1014 cases. Radiology. 2020 Feb 26:200642. doi: I0.I I 48/radiol.2020200642

2I. Wang D, Hu B, Hu C, Zhu F, Liu X, Zhang J, Wang B, Xiang $\mathrm{H}$, Cheng Z, Xiong Y, Zhao Y, Li Y, Wang X, Peng Z. Clinical Characteristics of 138 Hospitalized Patients With 2019 Novel Coronavirus-Infected Pneumonia in Wuhan, China. JAMA. doi: 10.100I/jama.2020.1585

22. Pan F,YeT, Sun P, Gui S, Liang B, Li L, Zheng D, Wang J, Hesketh $\mathrm{RL}$, Yang L, Zheng C. Time course of lung changes on chest CT during recovery from 2019 novel coronavirus (covid-19) pneumonia. Radiology. doi: I 0.I I 48/radiol.2020200370

23. Shi H, Han X, Jiang N, Cao Y, Osamah A, Gu J, Fan Y, Zheng C. (2020) Radiological findings from 81 patients with COVID-19 pneumonia in Wuhan, China: a descriptive study. [online] thelancet.com 24 February 2020. Available at: https://www.thelancet.com/journals/laninf/article/PISI4 73-3099(20)30086-4/fulltext\#figures.https://doi.org/I0.1016 ISI 473-3099(20)30086-4 [accessed 25 February 2020].

24. Lee EYP, Ng M-Y, Khong P-L. COVID-19 pneumonia: what has $\mathrm{CT}$ taught us? [online] thelancet.com. Published:February 24, 2020. Available at: https:/www.thelancet.com/ journals/laninf/article/PIISI 473-3099(20)30I34-I/ fulltext\#\%20 DOI:https://doi.org/I0.I0I6/SI473-3099(20) 30I34-I
25. Wei Zhao, Zheng Zhong, Xingzhi Xie, Qizhi Yu, Jun Liu. Relation between chest CT findings and clinical conditions of coronavirus disease (COVID-19) pneumonia: A multicenter study. American Journal of Roentgenology. doi:I0.22 I4/AJR.20.22976 - Pubmed

26. Another Decade, Another Coronavirus. (2020). N Engl J Med. doi:10.1056/NEJMe200II 26 - Pubmed

27. Jeffrey $P$ Kanne, Brent $P$ Little, Jonathan H Chung, Brett $M$ Elicker, Loren $\mathrm{H}$ Ketai. Essentials for Radiologists on COVID-19: An Update-Radiology Scientific Expert Panel. (2020). Radiology. doi: I 0.I I 48/radiol.2020200527 - Pubmed

28. Inui Shohei, Akira Fujikawa and Motoyuki Jitsu et al. "Chest CT Findings in Cases from the Cruise Ship "Diamond Princess" with Coronavirus Disease 2019 (COVID-19)". Radiology: Cardiothoracic Imaging 2, no. 2 (2020): e200 I I 0. doi:I0.1 I48/ryct.2020200II0.

29. Bai HX, Hsieh B, Xiong Z, Halsey K, Choi JW, Tran TML, Pan I, Shi LB, Wang DC, Mei J, Jiang XL, Zeng QH, Egglin TK, Hu PF, Agarwal S, Xie F, Li S, Healey T, Atalay MK, Liao WH. Performance of radiologists in differentiating COVID-19 from viral pneumonia on chest CT. (2020). Radiology. doi: 10.1 | 48/radiol.2020200823 - Pubmed

30. Jinnon Zhang, Juquian Zhou, Yuqoing Yang, Wei Peng Wenjing Wang, Xuelin Chen. Therapeutic and triage strategies for 2019 novel coronavirus disease in fever clinics. Respiratory Medicine. Vol 8 March 2020. doi: https//.doi.org//0.1016/ s22I3-2600(20)3007I-0.

31. Yicheng Fang, Huangqi Zhang Jicheng Xie, Minjie Lin, Lingjun Ying, Peipei Pang, Wenbin Ji. Sensitivity of chest CT for COVID-19: Comparison to RT-PCR. Radiology. Feb 19 2020. doi: https://doi.org// 0.I I48/radiol.2020200432

32. D. Buonsenso,A. Piano, F. Raffaelli, N. Bonadia, K. De Gaetano Donati, F. Franceschi. Point-of-Care Lung Ultrasound findings in novel coronavirus disease- 19 pnemoniae: a case report and potential applications during COVID-19 outbreak. European Review for Medical and Pharmacological Sciences. 2020; 24: 2776-2780.

\section{CORRESPONDENCIA}

oguzman@auna.pe, dr.oscar.guzman@gmail.com

Fecha de recepción: 10-04-2020.

Fecha de aceptación: I5.04.2020.

CONFLICTO DE INTERÉS: Ninguno, según los autores. FINANCIAMIENTO: Por los autores. 